\title{
La tecnología adaptada: entre realidad $y$ el deseo
}

\author{
Joaquín Arriola
}

\section{LA DIFICIL DEFINICION DE LA TECNOLOGIA APROPIA- DA}

La tecnologia apropiada, tecnologia alternativa ó tecnologia intermedia (en adelante TA), surge como un intento de responder a las necesidades, deseos y recursos de quienes van a utilizarla. Generalmente, se define por oposición a la tecnología dominante, la desarrollada en los paises desarrollados capitalistas, que es el $90 \%$ de la comercializada a nivel mundial. Como señala Robert Solo (1966), las tecnologías "avanzadas", esto es, provenientes de los paises desarrollados:

"(...) han evolucionado en y consecuentemente se encuentran adaptadas a un entorno físico y social que difiere significativamente del de las sociedades en desarrollo. A causa de tales diferencias, su uso en la sociedad en desarrollo será en ocasiones antieconómico y técnicamente regresivo"

$$
\text { (R. Solo, } 1966 \text { p.91) }
$$

El modelo de desarrollo tradicional ha consistido en imitar el proceso de industrialización de los paises desarrollados, procurando fuertes inversiones en sectores altamente capitalizados, en industrias de bienes de consumo (industrialización sustitutivas de importaciones) o en industrias orientadas al mercado mundial.

En todos los casos, sin embargo, aparecen problemas originados por el tipo de tecnología que comporta el iniciar esos procesos de industrialización imitativa; las tecnologias diseñadas en sociedades desarrolladas requieren su asimilación por parte de los paises desarrollados, lo cual no siempre se produce en forma directa: 
"En la medida en que las tecnologias avanzadas no son directamente transferibles su asimilación requiere una intervención social y una competencia social: $1^{2}$ reconocer lo que puede ser transferido directamente y lo que puede ser adaptado para la transferencia, luego, en $2^{9}$ adaptar las tecnologías avanzadas para su aplicación en la economía de baja productividad, o en $3^{\circ}$ reestructurar el marco de operaciones para proveer un entorno más favorable para la tecnologia avanzada".

(A. Solo 1986 p. 92).

Pero los problemas no se situan solo en el contexto de asimilación o transferencia, sino una vez introducida la tecnología en el pais atrasado al utilizarse en contextos socioeconómicos para los que no ha sido disefrada:

"La maquinaria altamente capitalizadas, por ejemplo, reemplaza a menudo a técnicas tradicionales basadas predominantemente en el trabajo, con toda la infraestructura de aprendizaje que frecuentemente las rodea. También ha fracasado en lo que respeta a crear los puestos de trabajo que se necesitan para ir al ritmo el rápido crecimiento de la fuerza de trabajo, en gran parte debido al fuerte descenso de la mortalidad infantil. La falta de empleo. en las zonas urbanas de muchos paises subdesarrollados es actualmente el principal problema social y político.

La introducción de técnicas industriales avanzadas puede crear también una fuerte disminución en las reservas extranjeras. Estas son necesarias para pagar los intereses del capital prestado, para licencias y patentes y muy a menudo incluso para materias primas tales como los plásticos que se necesitan en el proceso de producción. Además los problemas del medio ambiente en amplia escala han sido muy a menudo el resultado de tecnologias y técnicas de ingeniería occidentales en países subdesarrollados, sin preocuparse gran cosa por las condiciones ambientales existentes.

Pero, quizá, de 10 que más se puede acusar a las políticas dedicadas a una rápida industrialización es de que han fracasado a la hora de resolver, y en muchos casos lo único que han conseguido ha sido exacerbar los problemas sociales de pobreza, desnutrición y bajos niveles de vida en general que indirectamente trataban de resolver".

(D. Dickson, 1978 pp. 133-134) 
La TA se concibe en un primer momento para evitar los problemas de los procesos de industrialización tardia que nos describe $D$. Dickson.

Resulta difícil dar una definición sencilla de lo que se entiende por TA. De la complejidad de tal intento, nos da cuenta la gran variedad de denominaciones que se utilizan para designar realidades similares. $\mathrm{N}$. Jéquier y G. Blanc (1983) han realizado el esfuerzo de diferenciar contenidos entre las diversas denominaciones.

-Tecnologia alternativa: "nuevos tipos de equipamiento y nuevas formas de organización que representan una alternativa viable a las nuevas tecnologias convencionales actualmente existentes".

-Tecnologia apropiada: "término genérico que recubre una vasta gama de tecnologias que poseen una o varias de las siguientes características: bajo coste de inversión por puesto de trabajo o por unidad producida, gran sencillez en el terreno operativo, grado elevado de adaptación a un entorno social o cultural dado, empleo racional de los recursos locales, producto final poco costoso, posibilidad de crear un gran número de empleos".

-Tecnología comunitaria: "tecnología de pequena talla que no precisa de estructuras complejas, que se situa a la medida de las necesidades y capacidades de pequeñas comunidades ubanas o rurales y que procura valorizar la participación comunitaria en el proceso de toma de decisiones".

-Tecnología dulce: "tecnología bien adaptada al entorno social y cultural local, que emplea recursos renovables más que recursos no renovables y que altera en escasa medida los ecosistemas del entorno".

-Tecnología Intermedia: "una tecnologia que se encuentra a medio camino entre la tecnología tradicional y la tecnología moderna. Este carácter intermedio es relativo (geográfica y sociohistóricamente)".

-Tecnologia poco costosa en capital (capltal-saving technology): "una tecnologia caracterizada esencialmente por el débil coste en capital y el bajo nivel de inversión requerida para crear un empleo".

Con todo, la diferencia que se pueda establecer entre las diversas denominaciones de la TA se enfrenta al escaso desarrollo que tiene todavia, en su aplicación efectiva. Siguiendo con la posible definición de la TA, J.P. Vigier ofrece la siguiente:

"La tecnologia apropiada es la posibilidad para un grupo de personas de elegir la técnica que conviene a sus necesidades de producción y de consumo, cuya posesión y evolución les resulta 
controlable y que les permite adquirir una relativa autonomía de vida y de gestión.

(J.P. Vigier 1982 p. 10)

Por su parte D. Morawetz de la siguiente definición:

"La tecnologia apropiada puede definirse como el conjunto de técnicas que realizan un uso óptimo de los recursos disponibles en un entorno dado. Para cada proceso o proyecto, se trata de la tecnología que maximice el bienestar social si los precios de los factores son fijados como precios sombra".

(D. Morawetz 1974 p. 517)

La definición de J. P. Vigier presenta la dificultad de que la existencia de diversos grupos en conflicto en el seno de una sociedad da lugar a que sus objetivos discrepen entre si, y por lo mismo, lo harán sus opciones tecnológicas. Resulta problemático afirmar que existe un conjunto de objetivos sociales que permita hablar de una tecnología apropiada para todos los grupos sociales. Por el contrario toda tecnologia es, según la definición de Vigier, tecnologia apropiada, pues siempre existe un grupo social cuyos intereses se encuentran mejor cubiertos con la tecnología disponible: los capitalistas multinacionales, las élites locales de uno u otro tipo etc...

La definición más "técnica" de Morawetz, tampoco es plenamente satisfactoria. En esta definición se considera como un dato el conjunto de técnicas disponible, considerando que solo existe un único conjunto de precios sombra que permita la selección óptima de entre las técnicas disponibles.

En la realidad, sin embargo, la existencia de grupos sociales son intereses diversos no permite hablar de un óplimo único, y en consecuencia, tampoco de un único conjunto de precios sombra. Por otra parte, si el conjunto de técnicas disponible se considera que puede variar, resulta imposible definir un conjunto particular como "la tecnologia apropiada". En todo caso, si resulta siempre posible perfeccionar a partir de cualquier conjunto de técnicas dado la satisfacción de las necesidades sociales, nunca se podrá definir la tecnologia apropiada, y solo cabe hablar de unas más apropiadas que otras (lo mismo que unas resultarán más eficientes que otras) (F. Stewart 1977 p. 95).

Para comprender mejor la noción de TA es necesario delimitar el ámbito de aplicación de la misma. En efecto, es descartando las so- 
luciones extremas, que se ha llegado a la noción de TA: ni la autarquia tecnológica ni la industrialización salvaje han dado en la práctica los resultados deseados en lo que a desarrollo se refiere.

La autarquia tecnológica es inconcebible porque ningún pais, ni siquiera el más desarrollado, puede disponer de las capacidades técnicas y cientificas que le permitan cubrir todo el abanico de las posibilidades tecnológicas actuales. La RFA y el Japón son un buen ejemplo de ello, porque las partidas tecnológicas de sus balanzas de pagos han ido deteriorándose en el transcurso de los últimos años ( $P$. Judet 1981 p. 117). Por el contrario, el relativo fracaso de la industrialización importada y los trastornos socioeconómicos que ha suscitado en distintos países, muestra también los limites del crecimiento industrial por importación de tecnología.

Son precisamente estas constataciones, y no los refinamientos teóricos sobre uno u otro aspecto del subdesarrollo, las que han conducido a distintos organismos internacionales a abandonar ciertos prejuicios y a considerar la noción de tecnologia apropiada con interés y comprensión.

A menudo, la TA se ha definido a partir de la puesta en evidencia de la inadecuación de mucha de la tecnología de los paises desarrollados utilizada por los países del Tercer Mundo.

El carácter relativamente menos desarrollado de la periferia en relación a los paises del centro ha llevado a algunos investigadores a proponer el desarrollo de una tecnología vinculada a las condiciones sociales, no ya de los paises subdesarrollados, sino del seclor tradicional de estos paises. De ahí surge el concepto de "tecnología intermedia".

\section{LA "TECNOLOGIA INTERMEDIA". E.F. SCHUMACHER}

El nombre de "tecnologia intermedia" aparece por primera vez a mediados de los años 60 en los textos de E.F. Schumacher. Frente a la tecnología disenada, pensada para aumentar el rendimiento, elevar la productividad del trabajo, la tecnologia intermedia responde a la búsqueda prioritaria de otros objetivos, en particular "maximizar las oportunidades de trabajo para los parados y para que aquellos que tienen un empleo inadecuado para sus aptitudes".

La tecnologia intermedia trata de hacer frente al principal problema con que se encuentran los paises subdesarrollados, cual es el del desempleo masivo: "es necesario enfatizar que la necesidad básica consiste en nuevos puestos de trabajo, literalmente millones de nuevos puestos de trabajo" (EFS 1973 p. 151). La tecnología intermedia pretende generar puestos de trabajo en el sector tradicional, no capitalista, 
de la economia en función de cuatro criterios básicos: 1

$1^{2}$ La creación de puestos de trabajo debe localizarse en el entorno en que vive la gente, evitando el efecto de desplazamiento y concentración en las áreas metropolitanas.

$2^{\circ}$ El coste de la creación de cada empleo, en términos de inversión en capital, creación de infraestructura o importaciones, debe ser lo suficientemente bajo como para permitir la creación de un número elevado de puestos de trabajo.

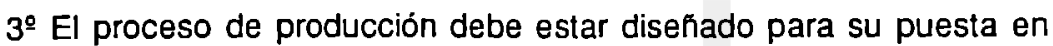
marcha por parte de trabajadores con un bajo nivel de cualificación.

$4^{2}$ La producción debe utilizar un elevado porcentaje de inputs de origen local y debe destinarse preferentemente a satisfacer las necesidades de consumo locales ${ }^{2}$.

A partir de estos criterios podemos identificar 3 fuentes principales de tecnología intermedia:

-El desarrollo de la producción tradicional indigena y de las técnicas de servicios.

- La adaptación de las tecnologías que provienen de los $P D$, reduciendo la escala y el coste de la inversión de funcionamiento y puesta en marcha, modificando la relación capital-trabajo y el tipo de inputs requeridos para hacer mayor uso de los recursos locales.

- Investigación y desarrollo de técnicas intermedias nuevas, en función de las necesidades de la zona en que se vayan a implementar.

El propio Schumacher reconoce que la tecnologia intermedia como modelo más adecuado, lo es en referencia a! sector "no moderno" de los países subdesarrollados:

"Me propongo aqui exclusivamente el problema de ayudar a la gente en el sector no moderno. Esto no implica la sugerencia de que el trabajo constructivo en el sector moderno debe abandonarse y es evidente que en cualquier caso continuará. Pero lo que sí supone es la convicción de que todo éxito en el sector moderno ha de ser inútil, salvo que también haya un crecimiento saludable (...) entre los numerosos grupos de gente cuya vida está caracterizada no solo por una extremada pobreza, sino también por una total desesperanza".

(E. F. Schumacher 1973 p. 149) 
Esta perspectiva le lleva a poslular que la tecnologia intermedia, con las caracteristicas y condiciones anteriormente enunciadas, se concentre en las áreas rurales y pequeñas poblaciones, al objeto de generar una "estructura agro-industrial".

El modelo que postula la tecnología intermedia no pretende sustituir a la aplicación de otros modelos tecnológicos en todos los campos de la producción, pero si se presenta como la más adecuada para satisfacer las necesidades de la mayor parte de la población de los PSD; la tecnologia intermedia pretende desarrollar las herramientas de escala adecuada que permitan cubrir las necesidades elementales de las masas pobres del Tercer Mundo, principalmente de las ubicadas en el sector tradicional de la economia.

\subsection{Los límites de la tecnologia Intermedia}

Se puede considerar que la tecnologia intermedia forma un subgrupo dentro de lo que generalmente se entiende por TA, debido a su referencia exclusiva a las necesidades sociales del sector no modernizado o no capitalista de los paises de la periferia. Por ello no responde al criterio más amplio de lo que es la TA. Como indica J.P. Vigier:

"(...) la noción de tecnología apropiada puede y debe aplicarse tanto a los paises desarrollados como al Tercer Mundo. No se trata de técnicas atrasadas o incompletas, se trata de técnicas que corresponden a las necesidades reales de los hombres, que son elegidas por ellos y de las cuales se pueden apropiar insertándolas en su cultura y forma de vida. La técnica impuesta, es en cierta forma la tecnología democrática, pues es cierto que, como hemos podido comprobar en el debate sobre la energia nuclear, la elección tecnológica es también una elección política".

$$
\text { (J.P. Vigier } 1982 \text { p. 10) }
$$

Hay un cierta coincidencia entre la opinión de Vigier y algunos de los resultados de un informe de la Oficina de Evaluación Tecnológica del Congreso de los Estados Unidos:

"Un creciente número de observadores han señalado que, aunque lo pequeño es hermoso, no siempre es sensato. En algunas tareas es posible reducir 0 descentralizar una tecnologia grande, pero srería utópico e indeseable suprimirla del todo. En este sentido la TA encarna el principio de selectividad al conjuntar (o crear) una combinación de tecnologías pequenas y grandes para satisfacer 
necesidades y circunstancias específicas (...)

(...) mucho de lo que se denomina TA es en realidad un tipo específico de tecnología avanzada, encaminada a aprovechar condiciones cambiantes en materia de recursos. Para adaptarse al medio económico actual, por ejemplo, la TA ha desembocado en muchas tencologias relativamente complicadas que aprovechan eficientemente la energia y los recursos materiales".

(Oficina de Evaluación Tecnológica 1982 pp. 77-78)

Por otra parte, a la tecnología intermedia se le acusa también de representar una forma de "capitalismo a pequena escala".

"...con todas las desigualdades y la injusta distribución de las recompensas sociales que esto conlleva inevitablemente".

(D. Dickson 1978 p. 135)

En el análisis de los países subdesarrollados resulta factible diferenciar entre dos sectores, uno avanzado y otro tradicional desde el punto de vista del bagaje tecnológico. Pero derivar de este hecho un planteamiento en términos de sociedad dual "à la" Lewis, oculta el hecho de que el sector tradicional se encuentra vinculado al sistema capitalista, pues el propio funcionamiento del modo de producción capitalista en los países periféricos exige el mantenimiento del sector tradicional como reserva de fuerza de trabajo y como proveedor de alimentos y otros bienes-salario a bajo coste, lo cual permite reproducir la fuerza de trabajo con unos niveles salariales muy reducidos en relación a los paises desarrollados.

En cuestión tecnológica, se parte de una situación altamente diferenciada también desde el punto de vista institucional y organizativo. En este sentido, la actuación debe eslar encaminada a aproximar los dos sectores de la economia en términos de inversión per cápita, productividad del trabajo, satisfacción de necesidades básicas etc. Por eso, el tipo de técnicas requerido, o su modificación, diferirá en función del sector al cual vaya asociada, no siendo siempre la más adecuada la vinculada con el concepto de tecnologia intermedia.

En fin, una definición de la tecnología reducida a los medios de producción, supone pasar por alto los problemas estruclurales, políticos y sociales, sin cuya resolución difícilmente se van a resolver los problemas de miseria de la mayoria de la población del Tercer Mundo: reparto de la propiedad agricola, organización política, peso socioeconó- 
mico de las firmas multinacionales, analfabetismo etc.

\section{REQUISITOS DE LA TECNOLOGIA APROPIADA}

Ya hemos sefialado anteriormente como de toda técnica se puede decir en un sentido o en otro que es más apropiada o menos apropiada, según los objetivos que se persiga (no da los mismos resultado unas técnica si lo que se persigue es maximizar los beneficios, que si lo que se pretende es maximizar el empleo de mano de obra dentro de unos limites de rentabilidad) y dependiendo también del contexto socioeconómico en que se implante o desarrolle esa técnica.

La definición de lo que sea una TA, o la tecnología "más apropiada", será siempre una definición dinámica y por tanto relativa.

Lo más sencillo es definir la TA por oposición a lo que es tecnologia inapropiada en los paises de la periferia esto es, la mayor parte de la desarrollada en los paises del centro.

Una consideración fundamental es la distinción entre el sector moderno y el tradicional de las formaciones sociales subdesarrolladas: ni los requisitos, ni los objetivos prioritarios coincidirán en uno y otro. Como se ve en el cuadro 1, las caracteristicas del sector (M)oderno no coinciden con las de los paises más atrasados entre los de la periferia, o incluso con el sector (T)radicional de aquellos que poseen un cierto grado de urbanización e infraestructura industrial.

Partiendo de las caracteristicas de uno y otro sector, los objetivos de una tecnologia más apropiada que la generalmente en uso pasan por el aumento en la utilización de inputs locales, de tecnologias que requieran una cualificación menos específica y compleja de la fuerza de trabajo -matizando según los sectores $M$ y $T$ y las actividades productivas-. tecnologias de fabricación de productos apropiados, entendidos estos como los que sirven para cubrir las necesidades básicas de la población: sin entrar en una exposición pormenorizada del debate en torno a lo que se entiende por "necesidades básicas", el primer requisito para su identificación es el cubrir las necesidades de supervivencia y reproducción de la población en el sector $\mathrm{T}$ en los paises más atrasados, o ampliar la demanda interna, el consumo de masas, en el sector $\mathrm{M}$.

Por fin, el elemento fundamental de una tecnologia más apropiada desde la perspectiva de los países subdesarrollados es la que tiene como objetivo prioritario la reducción del desempleo masivo, en consonancia con lo requerido por cualquier politica de desarrollo.

La situación de desempleo masivo se produce sobre todo en el sector moderno: las conurbaciones que rodean todos los centros industriales 
Característica

Objetlvo

(para paises muy pobres)

I/L reducida

Caracterlstica

Objetlvo aumentar las opor-
tunidades de empleo

escala grande o reducir los requisipequeña (prefte. tos de la función pequeña)

empresarial; aumentar el acceso de empresarios locales

simplicidad de uso reducir los requisiy reparación

productos apropiados

urbana y rural tos de cualilicación de la fuerza de trabajo

eliminar especificidades excesivas, aumento de estandarización, diversificación de los productos de consumo según las necesidades de la mayoria de la población, ampliación del mercado interno pequeña escala

simplicidad de or- minimizar la cualiganización, uso y ficación de la fuerreparación za de trabajo y de la actividad empresarial

productos

apropiados

ampliar las actividades del sector $T$ para cubrir más necesidades énlasis en rural

proveer oportunidades en los lugares en que se localiza la población vinculada al sector $\mathrm{T}$

uso de inputs locales estimular otras actividades locales uso de inputs producidos localmento (materias primas y maquinaria pacto local, mayor integración intersectorial, relleno de la matriz industrial, reducir la dependencia.

Fuente: F. Stewart 1977 p. 98 y elaboración propia. 
del Tercer Mundo y plantean problemas de gestión de las ciudades en materia de urbanismo, sanidad, escolarización etc., pero a un nivel más primario, suponen un grave problema de desempleo o subempleo estructural.

La necesaria ampliación del mercado interno pasa necesariamente por la generación masiva de empleos en el sector $M$. Frente a este reto, parece obvio que la tecnología industrial de los paises desarrollados resulta generalmente poco adecuada, pues la elevada inversión por empleo creado se enfrenta a un mercado de capitales suficiente, a menudo dependiente de los aportes exteriores -via préstamos o inversión extranjera.

La TA sería, en este caso, aquella que maximiza el número de empleos para un nivel existente de ahorro (capacidad de inversión), dentro de un umbral de rentabilidad determinado: esta condición de la TA en los países subdesarrollados no agota la significación de esta noción - pues en tal caso nos estariamos refiriendo al concepto de tecnologia intermedia de Schumacher-. Hoy, la TA, de centrarse originariamente en una problemática social específica (la de creación de empleo en el Tercer Mundo, ha ido derivando rápidamente hacia una problemática socioeconómica más general. Así, deberian ser consideradas TA solo las tecnologias afectadas de un nivel de productividad suficiente como para permitir la creación de un cierto excedente social destinado a alimentar el crecimiento de las fuerzas productivas necesario para la eliminación progresiva de la miseria y permitir la aceleración del desarrollo.

Con frecuencia se identifica el término de tecnología adaptada con aquellas técnicas que, obsoletas en los países del centro, mantienen su viabilidad económica en la periferia por la diferente disponibilidad y estructura de coste de los factores productivos. Esto lleva a considerar que la tecnologia adaptada es necesariamente una tecnologia "menos avanzada" que la disponible en los paises desarrollados.

Sin embargo, conviene diferenciar entre lo que puede ser obsolescencia por razones de ingeniería de lo que se considera obsolescencia económica (Loehr y Powelson 1982 p. 21). En este sentido, la TA no puede dejar de ser tecnologia eflciente. Lo que resulta apropiado en un determinado contexto socioeconómico puede no serlo en otro y no solo por la diferente dotación de factores y precios del mismo sino, lo que es más importante, porque los objetivos que se persiguen en uno $u$ otro contexto pueden ser diversos. Bajo el criterio capitalista de la maximización del beneficio, no cabe ciertamente la distinción que se establece con la TA, y el criterio de eficacia coincide 
con el de maximización del beneficio. Es cuando se planifican objetivos generales, cuando la TA puede ser definida como la que minimiza el coste social de perseguir tales objetivos. Así, un proceso que requiera más mano de obra puede ser más eficiente si el objetivo básico perseguido es la reducción del desempleo a corto plazo. Pero en términos generales, se tratará de tecnologías dinámicas, capaces de conciliar las exigencias de lo inmediato (empleo) con las de más largo plazo (crecimiento y desarrollo). Su intensidad en capital puede variar por tanto de forma considerable de un pais a otro (nuevos paises industriales $u$ otras economias menos desarrolladas), de una rama a otra (agricultura, siderurgia, industrias mecánicas...), o de un punto a otro de un mismo territorio (ciudad-campo).

En cualquier caso, esta definición que aquí se propone de TA no tiene nada que ver con el de tecnologías atrasadas o fuera de uso en los paises desarrollados, y tampoco tiene porqué ser ajena a la tecnología de vanguardia en determinado uso productivo. Por el contrario, el concepto de TA remite necesariamente a la crítica de los criterios más adecuados para establecer una estrategia de desarrollo.

\section{LA ADAPTACIÓN TECNOLÓGICA, REQUISITO DEL DESA- RROLLO}

La cuestión de la adaptación tecnológica va más allá de los debates sobre las virtualidades para el desarrollo de una tecnología apropiada o alternativa

"Los paises del Tercer Mundo no pueden remediar el carácter inadecuado de la tecnologia -inadecuación resultante sea de la intensidad de los factores, sea del tipo de producto fabricado- más que determinando a su nivel el tipo de técnica adecuada a sus condiciones especíticas y estableciendo los mecanismos necesarios que garanticen la realización de esos objetivos".

(A. Sid-Ahmed 1977 p. 698)

La adaptación tecnológica es un requisito ineludible para toda formación social dinámica, y tendrá mayor importancia cuanto más retrasada se encuentre esta desde la perspectiva de la existencia y control de una base tecnológica de reproducción y desarrollo industrial. Se requiere una actividad de I+D destinada a adaptar el equipo sencillo, en particular equipo que se adapte a las condiciones locales del pais receptor. Asi, la tecnología agrícola tiene que adaptarse a las condiciones geográficas y climáticas; un gasto de $1+D$ para adaptar 0 
mejorar el diseño de un arado de tracción animal puede provocar importantes ganancias de productividad. El establecimiento de disefios apropiados puede facilitar la difusión de nuevas técnicas producidas. En particular en los países más atrasados, la actividad fundamental de I+D y de los esfuerzos del gobierno en esta materia, deberán ir orientados hacia este tipo de actividad adaptativas, más que hacia el descubrimiento de técnicas y procesos enteramente novedosos. Como se indica en un estudio de la CNUCED (1985 p. 164), hasta ahora el objeto de la mayoria de las actividades de disefio en el sector industrial de los países subdesarrollados ha sido la adaptación de los disefios importados a las condiciones locales, en materia de requerimientos de la demanda interna, la disponibilidad de componentes y el mayor uso de materias locales para reducir la dependencia de las importaciones.

La actividad de adaptación tecnológica es además una vía que facilita la apropiación por parte de la fuerza de trabajo técnica local de la tecnologia en uso. Con frecuencia la tecnologia disponible obtenida por importación (transferencia) se recibe en unas condiciones tales que impiden su difusión en el tejido productivo local, al existir dificultades para que se lleve a cabo un proceso de aprendizaje a partir del uso de la tecnología transferida: por ejemplo, la información disponibles sobre el sector de producción de bienes de capital en los paises de la periferia ${ }^{3}$ apunta como las licencias relativas a bienes de capital son utilizadas para adquirir el disefio básico de un productor y los diseños de detalles para fabricar piezas y componentes, o bien no se incluyen por regla general, al existir una capacidad autónoma para desarrollar dichos elementos (India), o se incluyen para justificar un pago más elevado por la licencia, aunque el concesionario no los necesite realmente (Brasil), o las empresas dependen totalmente de los concedentes de licencias, existiendo muy poca transferencia de tecnologia de diseño (Corea) o si se incluyen el diseno de detalles y la asistencia en la fabricación y la capacitación, no abarcan la metodologia del diseño básico (China). Los proveedores de tecnologia tienen interés en mantener la necesidad de los receptores de la misma de recurrir a ellos para asumir tareas de asistencia técnica, control de calidad u otras, que reproduzcan la dependencia tecnológica. La actividad de adaplación de la tecnología recibida, permite un conocimiento más riguroso de la misma que el que a menudo se puede desprender de los planos e instrucciones, facilitando asi el proceso de aprendizaje, dominio y difusión tecnológica.

Los países desarrollados, cuyas firmas poseen el control de la inmensa mayoria de las patentes y licencias, tienen un papel que jugar a la hora de facilitar a los paises del Tercer Mundo la apropiación de las tecnologias de producción que contribuya a la creación de la base 


\section{Cuadro 2}

El movlmiento de la tecnología apropiada en 1977: número de organizaclones, presupuesto y hombres mes de trabajo en tecnologia aproplada

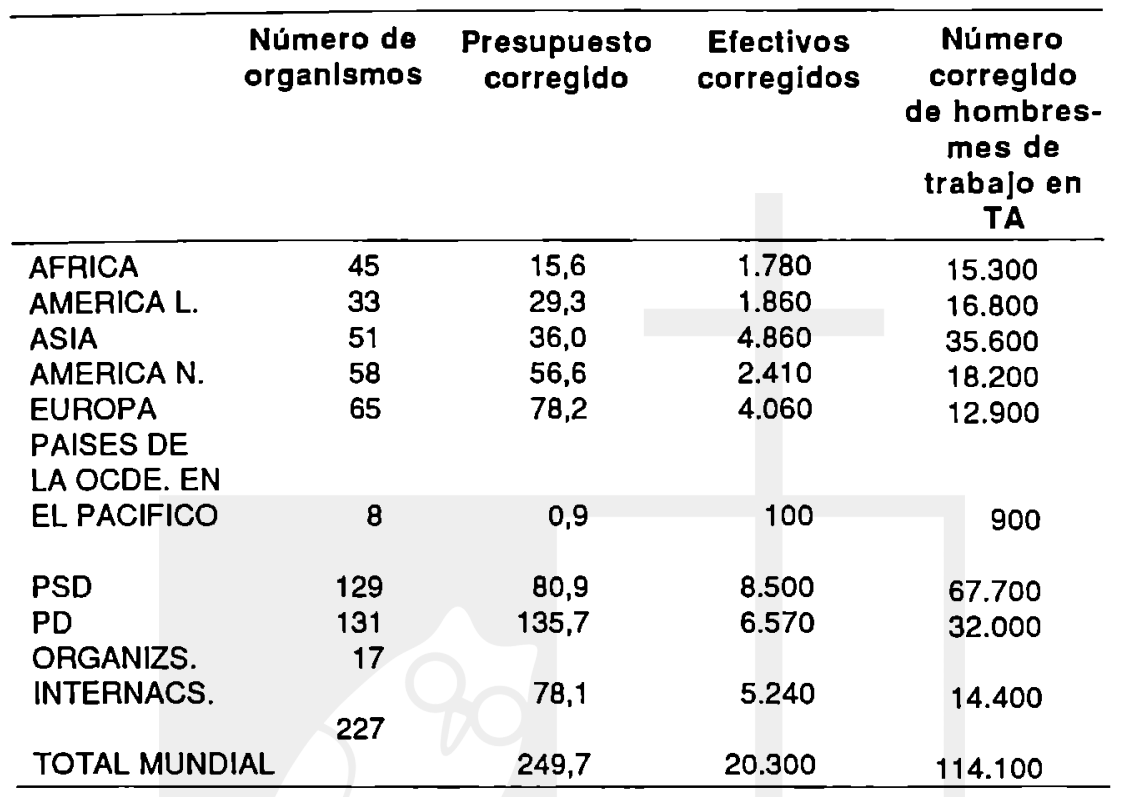

Fuente: Jéquier y Blanc 1983 p. 30.

industrial requerida en todo proceso de desarrollo.

\section{LA TECNOLOGIA ALTERNATIVA: SUS DIMENSIONES AC-} TUALES

El desarrollo de la I+D en materia de TA, aunque se realiza en la periferia en más de la cuarta parte del presupuesto mundial y alli se concentra el $60 \%$ de los efectivos humanos dedicados a la misma (cuadro 2), en los paises semiindustrializados del Tercer Mundo se carece por lo general de centros importantes de desarrollo de TA. Tal es el caso de Brasil, Corea del Sur, Argentina, Africa del Sur o Marruecos. Tan sólo México, India o Taiwán son excepciones a esta regla.

De 680 centros contactados, 142 se localizan en esta región. De 388 respuestas al cuestionario, 71 provienen de la misma zona. (De los EE.UU. y Europa Occidental, son 265 los organismos contactados y 163 las respuestas). Hay que tener en cuenta, además, que el $40 \%$ de las actividades de los centros de TA situados en los paises de la OCDE se 
encargan de problemas de los paises subdesarrollados.

Por lo que respecta a los diversos tipos de organismos que se ocupan de la TA, estos son entre los censados: Centros de Tecnologia Apropiada $(18,7 \%)$; Universidades $(15,7 \%)$; Otros centros de investigación $(24,1 \%)$; Industrias $(11,2 \%)$; Agencias gubernamentales $(14,1 \%)$; Organizaciones internacionales $(7,1 \%)$; Organizaciones financieras $(4,7 \%)$ y otros organismos $(4,4 \%)$.

Si nos fijamos en el cuadro 3 observamos que la principal actividad en materia de TA es la vinculada con la agricultura (508), seguida por la vinculada co la producción de energia (321). La TA vinculada con la industria (promoción de pequefa industria, construcción, artesanado) aparece más relegada (259). Es importante señalar que el sector energético es más importante en los países industrializados, pues en los países subdesarrollados adquieren más relevancia las actividades de TA industrial.

Es de constatar la escasa relevancia de la TA en materia sanitaria - tan solo la nutrición aparece entre las 21 principales actividades. Ello obedece, posiblemente, a que en los países subdesarrollados o es una actividad estatilizada, o está en manos de organismos internacionales como la OMS o la Cruz Roja - lo que a los efectos, es lo mismo: no se le otorga la relevancia que debería tener la propia iniciativa, individual y colectiva, de los "usuarios", a los que se les concede escasa autonomia en lo que respecta al cuidado de su salud- actividad, por otro lado, que representa un cierto tabú su tratamiento en tanto que actividad productiva y por tanto, sujeta a rendimientos monetarios.

Con todo, hay que subrayar que las actividades de TA son marginales en el conjunto de la actividad socioeconómica mundial. En 1977, la suma total destinada a la TA no llegaba ni al $1 \%$ de los recursos de $I+D$, representando menos del $0,02 \%$ del PIB mundial.

La I+D es la principal actividad de los organismos que se ocupan de la TA: aproximadamente el $27 \%$ del esfuerzo mundial en materia de TA consiste en I+D, seguida de información (19\%), difusión $(15 \%)$, educación (14\%) y las actividades de apoyo (12\%). Entre las actividades de segundo rango se cuentan los estudios de política general y la acción ante las autoridades políticas $(4 \%)$, la asistencia a otras organizaciones de TA (3\%) y un $2 \%$ destinado a la producción comercial. Esto último da ideade hasta que punto la TA es una actividad escasamente integrada en la actividad productiva, solamente un $5 \%$ de la actividad mundial de TA está subvencionada por la industria privada, ${ }^{5}$ mientras que las gobiernos financiaban directamente el $33 \%$ del presupuesto de los 
organismos de TA, elevándose al $50 \%$ el total de la financiación por el sector público y al $19 \%$ el de organismos privados sin fines lucrativos.

La TA conoce en la década de los 60 y los 70 un crecimiento considerable: de representar un volumen de gaslo de 620 millones de $\$$ en 1975, en 1977 era de 1.075 millones de $\$$ y en 1980 se trataria de una citra situada en torno a los 2.300 millones de $\$$.

Con todo, todavia es escaso el porcentaje de esos recursos destinados a la experimentación a la investigación o a los proyectos piloto.

\section{Cuadro 3}

Principales campos de actividad de los centros de tecnología alternativa

\begin{tabular}{|c|c|c|c|}
\hline \multicolumn{2}{|c|}{$\begin{array}{l}\text { en los palses } \\
\text { subdesarrollados }\end{array}$} & \multirow{2}{*}{$\begin{array}{c}\begin{array}{c}\text { en los palses } \\
\text { industrlalizados }\end{array} \\
24\end{array}$} & \multirow{2}{*}{$\frac{\text { Total }}{54}$} \\
\hline GANADERIA & 30 & & \\
\hline NUTRICION & 30 & 24 & 54 \\
\hline TRATAMIENTO DE AGUAS & 27 & 28 & 55 \\
\hline PROTECCION DE SUELOS & 31 & 26 & 57 \\
\hline ARTESANOS & 36 & 24 & 60 \\
\hline IRRIGACION & 37 & 24 & 61 \\
\hline $\begin{array}{l}\text { PAODUCCION DE METANO } \\
\text { ELIMINACION DE }\end{array}$ & 28 & 34 & 62 \\
\hline BASURAS DOMESTICAS & 28 & 35 & 63 \\
\hline NUEVOS CULTIVOS & 33 & 31 & 64 \\
\hline ENERGIA HIDRAULICA & 32 & 33 & 65 \\
\hline ENERGIA EOLICA & 29 & 49 & 71 \\
\hline MAOUINAS AGRICOLAS & 37 & 36 & 73 \\
\hline FORMACION DE ADULTOS & 48 & 30 & 78 \\
\hline ECONOMIA DE ENERGIA & 29 & 49 & 78 \\
\hline HERRAMIENTAS AGRICOLAS & 50 & 31 & 81 \\
\hline RECURSOS DE AGUA & 43 & 42 & 85 \\
\hline CONSERVACION Y TRATA- & & & \\
\hline MIENTO DE COSECHAS & 46 & 39 & 85 \\
\hline TECNICAS DE CULTIVO & 50 & 40 & 90 \\
\hline $\begin{array}{l}\text { MATERIALES DE CONSTRUC. } \\
\text { PROMOCION DE LA }\end{array}$ & 50 & 42 & 92 \\
\hline PEQUENA INDUSTRIA & 62 & 45 & 107 \\
\hline ENERGIA SOLAR & 45 & 71 & 116 \\
\hline
\end{tabular}

Fuente: Jéquier y Blanc 1983. 
La elevada importancia de los recursos destinados a intercambio de nformación, indica que la TA se encuentra todavía en sus primeras etapas.

Esto se refleja claramente en las escasas vinculaciones de los organismos de TA con el sistema de inversiones privadas. Hay una gran dificultad práctica en la comercialización de las innovaciones tecnológicas de este tipo.

La TA ha estado -y sigue estando en gran medida vinculada a una crítica ideológica y cultural a la evolución social y tecnoeconómica del sistema vigente. La TA vincula a menudo su propio desarrollo a la crítica a los fundamentos del sistema, al primado del beneficio, al beneficio financiero como criterio de eficacia económica, a la ideología desarrollista, al mito del crecimiento. Todo ello hace que con frecuencia las empresas no tomen en consideración los desarrollos tecnológicos vinculados a la TA, por considerarlos a priori no rentables, o en todo caso, ajenos a su estrategia empresarial.

Por otro lado, en la medida en que los grupos de TA no pueden reforzar su capacidad de investigación, de ensayo y puesta punto de sus innovaciones; en la medida en que no sean capaces de transferir su trabajo y conocimientos hacia las instituciones que poseen la experiencia y el personal adecuado - en particular las empresas industriales ${ }^{6}$ y los centros de investigación convencionales, la TA no dará el salto de convertirse en un criterio general de la política de $1+D$ en materia tecnológica, manteniendo su orientación fuertemente sesgada hacia la resolución de problemas específicos, sobre todo de los países subdesarrollados, en el sector "tradicional" o "atrasado".

"(...) las ideas aportadas por el movimiento de la Tecnologia Alternativa han penetrado mucho más profundamente y a niveles más elevados, de lo que tienen tendencia a creer los críticos de la Tecnologia Alternativa. Por el momento, por supuesto, las realizaciones prácticas de esta revolución son todavia modestas y no tienen la dimensión suficiente para rivalizar con el sistema tecnológico existente tal como lo conocemos actualmente. El factor más importante es que esta revolución subestima lo que podria ser un cambio radical de la demanda cultural en materia de tecnologia, la tecnología es el producto de una cultura y su evolución se encuentra menos determinada por lo que es técnicamente posible que por aquello que resulta socialmente aceptable y culturalmente deseable. El movimiento de la Tecnología Alternativa de hecho, conscientemente 0 no, ha permitido cristalizar una demanda social 
en favor de nuevos tipos de tecnología que exigen menos capital, tienen una talla más reducida, ahorran recursos naturales y se adaptan mejor al hombre y a su entorno cultural. El sistema tecnológico representado por las grandes empresas industriales, los centros de investigación convencionales, los organismos de financiación, las agencias de planificación y los establecimientos de enseñanza, no se han adaptado todavia a esta nueva demanda. Este cambio requiere tiempo, e incluso mucho tiempo, no a causa de la mala voluntad, la ceguera o la incompetencia de los participantes, sino tan solo por el amplio retraso que existe entre la aparición de una nueva demanda y su satisfacción por la aparición de los productos o servicios nuevos. En la actual situación, lo que ofrece el sistema tecnológico es fundamentalmente inadaptado a las exigencias de la sociedad y la amplitud de esa diferencia proviene, en gran parte, de la rapidez del cambio de la demanda cultural y tecnológica en los últimos afios".

(Jéquier y Blanc 1983 p. 185)

\section{LA DIFUSIÓN DE LAS INNOVACIONES EN TECNOLOGÍA ALTERNATIVA}

Prácticamente la totalidad de las organizaciones de TA dedican gran parte de su tiempo y esfuerzos a difundir sus equipos y desarrollos tecnológicos. Incluso tenemos el caso de aquellas organizaciones cuya única tarea es precisamente difundir información, impulsar el uso de nuevas técnicas y tecnologias alternativas, en los diversos dominios de la actividad pública y empresarial.

Entre los obstáculos a la difusión de las innovaciones que citan las organizaciones de TA, la limitación financiera aparece sin duda como el más reconocido. No se trata estrictamente de la falta de dinero sino, más bien, el localizar proyectos interesantes, desarrollar las capacidades organizativas y de gestión y mejorar la competitividad de la TA frente a otro tipo de tecnologias modernas y tradicionales. Todas estas tareas exigen inversiones importantes, que a menudo no se pueden llevar a cabo por no disponer de los créditos suficientes.

Las relaciones institucionales de las organizaciones de TA son en su mayor parte con organismos públicos: universidades, administración, organizaciones internacionales.

Con frecuencia los proyectos, las innovaciones de TA se enfrentan a la lentitud, a la desconfianza frente a las soluciones originales, a los aparatos burocráticos de las instituciones públicas. La burocracia se les 
presenta así como el segundo obstáculo en importancia a la difusión de las TA.

Las reticencias a aceptar las innovaciones no es un obstáculo que planteen solo los organismos públicos. Con frecuencia, los propios destinatarios de la innovación se muestran reticentes a abandonar sus métodos e instrumental tradicional: incluso, a la hora de elegir entre una técnica de tipo alternativo y otra tradicional o moderna, la decisión se inclina por esta última, aun cuando todos los argumentos de tipo socioeconómico aconsejen la elección de la primera, a causa de la presión que implia la publicidad o los lugares comunes, como que las mejores soluciones son las más costosas.

A continuación, aparecen las dificultades de tipo "interno": falta de competitividad frente a las tecnologias modernas, falta de apoyo técnico $\longrightarrow$ las citadas en los lugares $11^{\circ}$ y $12^{9}$ por las organizaciones de TA, dificultades de mantenimiento y de personal cualificado. De todas ellas, la primera indica que la sustitución de una técnica o tecnologia tradicional por una TA es más difícil si existe una tecnologia moderna capaz de realizar esa misma función. Esa falta de competitividad frente a la tecnología moderna no es ajena a los otros factores citados, pero va má allá: la TA se tiene que enfrentar no solo con el rendimiento comparativo de la tecnología moderna, sino con el conjunto de valores sociales, con la nueva sociedad emergente, con la posibilidad de aproximarse a la riqueza de los paises industrializados, que anuncia la tecnologia moderna. Una de las mayores dificultades de la TA es precisamente el ir cargada de valores "contraculturales", un obstáculo vital a la difusión de una propuesta en un medio dominado por los valores de la cultura hegemónica - la de los países desarrollados.

Podemos clasificar en tres grandes grupos los obstáculos a la difusión de las innovaciones en TA, recogiendo entre paréntesis el lugar que ocupan, según las respuestas al cuestionario de la OCDE:

-Dillcultades Instltucionales: falta de créditos (1), burocracia (2), actitudes de los dirigentes políticos (7) este obstáculo es citado en tercer lugar por las organizaciones de TA de los paises desarrollados y en séptimo por las de los países subdesarrollados-, legislación inadecuada (8).

-Diflcultades sociales: rechazo a la aceptación de las innovaciones (3), inaceptabilidad social o cultural (6), falta de fiabilidad (10), falta de motivación de los usuarios (14).

-Dificultades técnlcas: falta de competitividad frente a las tecnologias modernas (4) y tradicionales (9), falta de apoyo técnico (5), 
dificultades de mantenimiento (11), falta de personal cualificado (12), falta de tiempo (13), falta de información o contactos (15).

A la vista de estas valoraciones, se puede pensar que existen buenas razones para creer que la mayoria de las organizaciones de TA tienen tendencia a subestimar el papel que juegan los factores técnicos y olvidar que las tecnologias alternativas precisan de un largo recorrido antes de poder situarse al nivel de las tecnologias más comunes, que pasaron su prueba hace ya mucho tiempo y poder convertirse en una alternativa válida frente a ellas.

\section{CONCLUSIONES}

Toda tecnología en uso implica algún grado de adaptación, en un sentido u otro, bien para obtener un rendimiento financiero adecuado a las condiciones estructurales de la formación social en que se implementa, bien para lograr objetivos no estrictamente vinculados a la lógica del capital, como puedan ser la maximización del empleo, la minimización de los insumos importados, el aprendizaje por parte de los usuarios (su adaptación progresiva al uso de tecnologías modernas) u otros.

Resulta imposible analizar los modelos de desarrollo tecnológico al margen de las características sociales y políticas del estudio de los casos específicos. Los patrones tecnológicos de los paises responden a una concreta inserción en la división internacional del trabajo, asi como a las características específicas nacionales del proceso de valorización social del trabajo: dinámica de los diversos agentes sociales, estructuración de los procesos de trabajo y de intercambio mercantil, etc.

La planificación tecnológica es un requisto del desarrollo, aunque tiene manifestaciones complejas, que van del facilitar la asunción por parte de la fuerza de trabajo de las capacidades de producción y reproducción de las tecnologías en uso, hasta la política de sustitución tecnológica en orden a maximizar el bienestar social, suprimiendo total o parcialmente la subordinación a los criterios de rentabilidad financiera capitalista.

La adaptación tecnológica no es exclusivamente un proceso técnico, sino también social, lo cual implica tanto la reconversión de las tecnologias disponibles cuanto un nuevo proceso de regulación social (formación de la fuerza de trabajo, modificación en las normas de consumo $y$ en el funcionamiento de los agentes sociales -Estado, sindicatos, empresarios etc.

La adaptación tecnológica no es exclusivamente un proceso técnico, 
sino también social, lo cual implica tanto la reconversión de las tecnologias disponibles cuanto un nuevo proceso de regulación social (formación de la fuerza de trabajo, modificación en las normas de consumo y en el funcionamiento de los agentes sociales -Estado, sindicatos, empresarios etc.). Son las políticas concretas las que llevan a situar el acento en uno u otro aspecto de la adaptación.

De lo expuesto se deduce que existe una distinción básica, teórica y práctica, entre la adaptación tecnológica y la tecnología alternativa, sustitutiva de tecnologias o patrones tecnológicos en uso, modernos o tradicionales.

Finalmente, el estatuto teórico de la tecnologia sigue siendo ambiguo, fluctuando entre su consideración como mercancia, como componente dinamizador de las fuerzas productivas y la valorización del capital, o como factor de reproducción/modificación social. Hay un campo importante abierto a la investigación teórica, que debe clarificar como puede y debe incidir el factor tecnológico en el diseño de las políticas de desarrollo.

\section{NOTAS}

1. Veamos como identifica Schumacher el principal problema de este modelo tecnológico:

No hay nada en la experiencia de los últimos 25 años que sugiera que la tecnología moderna, como nosotros la conocemos, pueda realmente ayudarnos a aliviar la pobreza del mundo, para no mencionar el problema del desempleo, que ya alcanza niveles del 30 por ciento en muchos de los llamados paises en desarrollo, y que ahora amenaza con convertirse en endémico también en muchos de los países ricos (...) asíque lo mejor sería enfrentarnos con el problema de la tecnologia. ¿Qué es lo que hace y que es lo que debería hacer? ¿Podemos desarrollar una tecnologia que realmente nos ayude a resolver muchos problemas, una tecnologia con rostro humano?"

(E.F. Schumacher 1973, p. 130)

2. Ver D. Dickson 1978 pp. 135-136. La definición que da Schumacher de "tecnología intermedia" queda bien ilustrada en el siguiente párrafo.

"Como dijera Gandhi, los pobres del mundo no pueden ser ayudados por la producción en masa, sino solo por la producción hecha por las masas. El sistema de producción masiva basado en una tecnologia sotisticada intensiva en capital, con una dependencia energética alta y ahorradora de mano de obra, presupone que ya se es rico, porque para establecer un sólo puesto de trabajo se necesita una cantidad considerable de inversión de capilal. El sistema de producción por las masas moviliza los recursos 
inapreciables que poseen todos los seres humanos, sus cerebros inteligentes y sus manos habilidosas y los apoya con herramientas de primera clase. La tecnologla de la ecológicamente dañina, autodestructiva en términos de recursos no renovables y embrutecedora para la persona humana.

La tecnología de la producción por las masas, haciendo uso de lo mejor del conocimiento y experiencia modernos, conduce a la descentralización, es compatible con las leyes de la ecología, es cuidadosa en su uso de los recursos escasos y se adapta para servir a la persona humana en lugar de hacerla sirviente de las máquinas. Yo la he denominado 'tecnologia intermedia para dar a entender que es muy superior a la tecnología primitiva de épocas pasadas, pero al mismo tiempo mucho más simple, más barata y más libre que la supertecnología de los ricos. Se la podria llamar también tecnología democrática o tencología del pueblo."

"Tal tecnología intermedia serla inmensamente más productiva que la tecnologia nativa (que a menudo está en decadencia), pero sería también mucho más barata que la tecnologia sofisticada, de alta intensidad de capital, de la industria moderna".

\section{(E. F. Schumacher 1983 p. 135 y 156)}

3. Con respecto a la cuestión del diseño y la fabricación de bienes de capital, la CNUCED realizó una encuesta sobre los principales productores de bienes de capital de Brasil, India, China y Corea del sur:

-Zhang Ren-yu: "Problemas de tecnología en el sector de bienes de capital: la experiencia de la A. P. China" (UNCTAD D/TT/57)

- "Problemas de tecnología en el sector de bienes de capital: estudio monográfico de los principales productores de maquinaria industrial del Brasil" (TD/B/C.6/AC.7/6 y corr. 1).

"Problemas de tecnologia en el sector de bienes de capital: estudio monográfico de los principales productores de maquinaria industrial de la India" (UNCTAD/TT/55)

4. Jéquier y Blanc (1983 p. 163) nos ofrecen otra clasiticación, en este caso referida a los organismos que respondieron a la encuesta: Organismos gubernamentales (34\%) Universitarias $(29 \%)$, Organizaciones religiosas $(21 \%)$, con fines lucrativos $(14 \%)$, sin fines lucrativos $(32 \%)$, Organizaciones internacionales No Gubernamentales (13\%), de la ONU (11\%), otras (3\%). (De los 277 organismos que participaron en la encuesta, unos 60 se agrupan en varios apartados).

5. En Africa y en América Latina, este porcentaje es aun menor. En Atrica, es relativamente más importante la ayuda extranjera y la gubernamental, en América Latina, la ayuda extranjera y la financiación de la Banca BID y otros-.

6. Solo 9 empresas industriales aparecen en la muestra analizada de 277 organismos vinculados a la TA. 


\section{REFERENCIAS}

CNUCED (1985): El Sector de Bienes de Capltal en los Paises en Vlas de Desarrollo. Naciones Unidas, Nueva York 1985234 pp. UNCTAD/TT/ 78.

DICKSON, David (1974): Tecnologla Alternativa y Polltlcas del Cambio Tecnológlco. H. Blume, Madrid $1978196 \mathrm{pp}$.

JEQUIER, Nicolás y BLANC, Gérard (1983): La Technologie Appropriée dans le Monde. Centro de Développement OCDE, Paris 1983239 pp.

JUDET, Pierre (1981): Lew Nouveaux Pays Industrlels. Les Editions Ouvrieres, Paris $1981174 \mathrm{pp}$.

LOHER, William y POWELSON, John P. (1982): "La función de la tecnologia: Análisis" en Perspectlvas Económicas № 4/1982 pp. 20-25.

MORAWETZ, D. (1974): "Employment implications of Industrialisation in Developing Countries: a Survey" on Economlc Journal, vol. 34 № 335. sept. 1974 pp. 491-542.

OFICINA DE EVALUACION TECNOLOGICA (1982): "Cambia el Concepto de Tecnologia Apropiada" en Perspectlvas Económicas, Congreso de los EE.UU. de A. Washington D.C. N² 2/1982 pp. 77-79.

SCHUMACHER, E.F. (1973): Lo Pequeño es Hermoso. H. Blume, Madrid $1978310 \mathrm{pp}$.

SID-AHMED, A. (1977): "L'Internationalisation de la Production et le Développement des Pays du Tiers-Monde" en Economles et Socletés vol. XI N 4 abril 1977 pp. 663-714.

SOLO, Robert (1966): "The Capacity to Assimilate an Advanced Technology" en American Economlc Revlew Papers \& Preceedings, mayo 1966 pp. 91 97.

STEWART, Frances (1977): Technology and Underdevelopment. MacMillan Press, Londres 1978304 pp. (existe trad. esp. en FCE)

VIGIER, Jean-Paul (1982): "La Technologie: Facteur du Développement?" en Dosslers Falm-Développement, agosto-sept. 1982 pp. 7-10. 